Revista Arbitrada Interdisciplinaria KOINONIA

Año VI. Vol VI. N³. Edición Especial: Educación II. 2021

Hecho el depósito de Ley: FA2016000010 ISSN: 2542-3088

FUNDACIÓN KOINONIA (F.K). Santa Ana de Coro. Venezuela.

Milagros Yovana Muñoz-Cabana

http://dx.doi.org/10.35381/r.k.v6i3.1436

\title{
Autorregulación del aprendizaje y rendimiento académico de los estudiantes
} universitarios

\section{Self-regulation of learning and academic performance of university students}

\author{
Milagros Yovana Muñoz-Cabana \\ mmunozc1@unmsm.edu.pe \\ Universidad Nacional Mayor de San Marcos, Lima \\ Perú \\ https://orcid.org/0000-0003-4734-7418
}

Recepción: 10 de junio 2021

Revisado: 15 de julio 2021

Aprobación: 01 de septiembre 2021

Publicación: 15 de setiembre 2021 


\title{
RESUMEN
}

La investigación tuvo por objetivo determinar la relación entre autorregulación del aprendizaje y rendimiento académico de los estudiantes universitarios en Lima Metropolitana. Se trabajo bajo un tipo de investigación descriptiva correlacional, apoyándose en un diseño no experimental. El resultado evidenciado en el estadígrafo Rho de Spearman, evidencia una correlación positiva baja o débil (Rho $=0,285)$. La significancia es menor que $0,05(0,000<0,05)$ por lo que se rechaza la hipótesis nula y se acepta la hipótesis alternativa; en este sentido, existe relación entre el aprendizaje autorregulado y rendimiento académico. Se advierte que es necesario promover desde el AA, la autoeficacia académica, siendo un sub proceso que conduce progresivamente a que el estudiante, adopte con mayor responsabilidad su aprendizaje.

Descriptores: Aprendizaje en línea; aprendizaje activo; método de aprendizaje. (Palabras tomadas del Tesauro UNESCO).

\begin{abstract}
The objective of the research was to determine the relationship between self-regulation of learning and academic performance of university students in Metropolitan Lima. It was worked under a type of correlational descriptive research, relying on a non-experimental design. The result evidenced in the Spearman Rho statistic shows a low or weak positive correlation (Rho $=0.285)$. The significance is less than $0.05(0.000<0.05)$ so the null hypothesis is rejected and the alternative hypothesis is accepted; In this sense, there is a relationship between self-regulated learning and academic performance. It is noted that it is necessary to promote academic self-efficacy from AA, being a sub-process that progressively leads to the student adopting the learning of it with greater responsibility.
\end{abstract}

Descriptors: Electronic learning; activity learning; learning methods. (Words taken from the UNESCO Thesaurus). 


\section{INTRODUCCIÓN}

El aprendizaje autorregulado (AA), es un proceso mediante el cual, el estudiante auto regula los procesos de cognición, metacognición, motivación, entre otros sub procesos complejos inherentes al cerebro y sistema nervioso central (Vázquez \& Daura, 2013); por consiguiente, el discente promueve un acercamiento con el aprendizaje en la conformación de una conducta comprensiva de la realidad, aunado a preocuparse por desarrollar habilidades para abordar tal contexto social (Daura, 2015); mientras que Pintrich, (2000), indica que este modelo, tiene las siguientes fases:

a) Fase 1. Previsión, planificación y activación: conlleva la programación, el establecimiento de metas de aprendizaje, así como considerar el propio conocimiento, las características del contexto y los saberes que se poseen sobre las tareas académicas a realizar.

b) Fase 2. Monitoreo: permite activar una mayor conciencia metacognitiva con el fin de realizar un seguimiento de las cuatro áreas mencionadas y la influencia que ejercen sobre el rendimiento académico.

c) Fase 3. Control: permite controlar y regular el propio comportamiento, el contexto y la tarea que se realiza.

d) Fase 4. Reflexión y reacción: posibilita evaluar el propio comportamiento, el contexto y la tarea, para posteriormente "reaccionar" y modificar algún aspecto que no favorece el propio aprendizaje

De ese modo, se evidencia que es un proceso sistemático, transitivo desde menor a mayor complejidad en el accionar por parte del estudiante, se requiere una adecuada maduración cognitiva para asumir con eficiencia este sistema de regulación del conocimiento, por cuanto, una persona que carezca de la motivación y responsabilidad necesaria, se verá reducida en aplicarlo correctamente, prefiriendo quedar en un rendimiento académico basado en el espectro clásico de la educación. 
El rendimiento académico, es un indicador que evidencia el desempeño cuantitativo cualitativo que ha logrado un estudiante en un determinado período de tiempo, este puede ser producto de varios factores intervinientes como lo socio económico (Rodríguez-Rodríguez \& Guzmán-Rosquete, 2019); sin embargo, suele depender de las prácticas pedagógicas desarrolladas para incentivar un adecuado aprendizaje, como en este caso, se relaciona el AA.

Por lo que cada estilo de aprendizaje podría influir en mayor o menor proporción en la generación de un óptimo rendimiento académico (Chambi-Choque et al. 2020), es por tal motivo que en la actual investigación, se procuró implementar el AA en estudiantes universitario de la ciudad de Lima - Perú, con la intención de conocer la relación entre ambas variables, de ese modo, se gestionaron resultados en favor de favorecer el uso de AA por cuanto contribuye al pensamiento crítico - creativo - innovador, que debe poseer un discente a nivel de instituciones universitarias.

Partiendo de lo expuesto, la investigación tuvo por objetivo determinar la relación entre autorregulación del aprendizaje y rendimiento académico de los estudiantes universitarios en Lima Metropolitana.

\section{METODO}

Se trabajo bajo un tipo de investigación descriptiva correlacional, apoyándose en un diseño no experimental (Hernández et al. 2014). La población estuvo conformada por 136 estudiantes de educación universitaria de 3 universidades privadas ubicadas en Lima Metropolitana - Perú; aplicándoseles dos instrumentos, uno dirigido a medir AA y otro para rendimiento académico. El instrumento 1, constaba de 19 ítems y el instrumento 2, configurado en 21 ítems, ambos configurados en tipo escala de Likert, pudiéndose conocer las percepciones de los estudiantes, sobre el comportamiento de las variables de estudio. 


\section{Confiabilidad del instrumento}

La confiabilidad de los instrumentos fue desarrollada a través del juicio de tres expertos y cálculo de coeficiente Alfa de Cronbach, obteniéndose valores para instrumento 1, de 0,85 e instrumento 2, 0,79 siendo considerables confiables para su aplicación en razón de los valores propuestos por (George \& Mallery, 2003), las cuales se puede apreciar en el cuadro 1.

\section{Cuadro 1.}

Criterio general para interpretar el coeficiente Alfa de Cronbach.

\begin{tabular}{ll}
\hline \multicolumn{1}{c}{ Coeficiente alfa } & \multicolumn{1}{c}{ Descripción } \\
\hline Coeficiente alfa $>.9$ & Excelente \\
Coeficiente alfa $>.8$ & Bueno \\
Coeficiente alfa $>.7$ & Aceptable \\
Coeficiente alfa $>.6$ & Cuestionable \\
Coeficiente alfa $>.5$ & Pobre \\
Coeficiente alfa $<.5$ & Inaceptable \\
\hline
\end{tabular}

Fuente: Tomada de George \& Mallery (2003, p. 231).

Como procedimiento de la investigación, se aplicó durante un semestre de estudios, el AA como estrategia en el ambiente de clases con los estudiantes, acotándose que se generó a través de la modalidad virtual de aprendizaje, constándose al final, que el rendimiento académico se encontraba en zonas favorables según el rango de puntuación de las universidades que participaron en la investigación, para tener en cuenta, los valores de la correlación, se trabajó con el contraste realizado a partir de los datos presentados en el cuadro 2. 


\section{Cuadro 2.}

Coeficiente correlacional de Spearman.

\begin{tabular}{|c|c|}
\hline Coeficiente & Tipo de Correlación \\
\hline$-<0.8$ a 1$]$ & Correlación negativa Muy alta o muy fuerte \\
\hline$-<0.6$ a 0.8$]$ & Correlación negativa Alta o Fuerte \\
\hline$-<0.4$ a 0.6$]$ & Correlación negativa moderada \\
\hline$-<0.2$ a 0.4$]$ & Correlación negativa Baja o débil \\
\hline$-<0$ a 0.2$]$ & Correlación negativa Muy baja o muy débil \\
\hline 0.00 & No existe correlación alguna entre variables \\
\hline$<0$ a 0.2$]$ & Muy baja o muy débil \\
\hline$<0.2$ a 0.4$]$ & Baja o débil \\
\hline$<0.4$ a 0.6$]$ & Moderada \\
\hline$<0.6$ a 0.8$]$ & Alta o fuerte \\
\hline$<0.8$ a 1$]$ & Muy alta o muy fuerte \\
\hline
\end{tabular}

Fuente: Hernández et al. 2014.

\section{RESULTADOS}

Los resultados se presentan en razón de los datos recopilados en la encuesta aplicada, analizados estadísticamente mediante el siguiente proceso:

\section{Cuadro 3.}

Relación entre Aprendizaje Autorregulado y Rendimiento Académico.

\begin{tabular}{llrr} 
& & $\begin{array}{c}\text { Aprendizaje } \\
\text { autorregulado }\end{array}$ & $\begin{array}{c}\text { Rendimiento } \\
\text { académico }\end{array}$ \\
\hline Rho de Spearman & Coeficiente de correlación & 1,000 &, $285^{* *}$ \\
& Sig. (bilateral) & 136 &, 001 \\
& $N$ & 136 \\
\hline$* *$
\end{tabular}

**. La correlación es significativa en el nivel 0,01 (bilateral).

El resultado evidenciado en el estadígrafo Rho de Spearman, evidencia una correlación positiva baja o débil (Rho $=0,285)$. La significancia es menor que $0,05(0,000<0,05)$ por lo que se rechaza la hipótesis nula y se acepta la hipótesis alternativa; en este sentido, existe relación entre el aprendizaje autorregulado y rendimiento académico. 
Revista Arbitrada Interdisciplinaria KOINONIA

Año VI. Vol VI. N³. Edición Especial: Educación II. 2021

Hecho el depósito de Ley: FA2016000010 ISSN: 2542-3088

FUNDACIÓN KOINONIA (F.K). Santa Ana de Coro. Venezuela.

Milagros Yovana Muñoz-Cabana

\section{Prueba de hipótesis}

H1: Existe relación entre autorregulación del aprendizaje y rendimiento académico de los estudiantes universitarios en Lima Metropolitana.

H0: No existe relación entre autorregulación del aprendizaje y rendimiento académico de los estudiantes universitarios en Lima Metropolitana.

Se acepta $\mathrm{H} 1$ y se rechaza $\mathrm{HO}$

\section{DISCUSIÓN}

Al existir relación entre las variables, se comprueba que el AA influye positivamente en el rendimiento académico, cuando este sea generado desde una concepción pedagógica por parte del docente en conformidad a lo establecido por (Barreto-Trujillo \& ÁlvarezBermúdez, 2020), entendiéndose que, para tal fin, lo primordial es fomentar la metacognición de forma efectiva en los estudiantes, dado paso progresivamente a la consecución de las fases planteadas por (Pintrich, 2000).

Por otro lado, (Alegre, 2014), advierte que es necesario promover desde el AA, la autoeficacia académica, siendo un sub proceso que conduce progresivamente a que el estudiante, adopte con mayor responsabilidad su aprendizaje, de ese modo; se proyecta un estudiante con mayor autonomía para afrontar los retos académicos, además de concatenarlos con experiencias de la vida social, influenciando positivamente en la generación de una persona en habilidad de ejecutar con mayor pertinencia, las habilidades propias de la profesión para la cual se forma.

Sin embargo, los resultados al ser una correlación positiva baja o débil, indica que se deben potenciar aspectos al AA como el auto concepto, motivación y estrategias pedagógicas, con la finalidad de incrementar el porcentaje de efectividad del AA sobre el rendimiento académico, lo cual concuerda con el estudio de (Mora-Yate et al. 2020). En ese sentido, (Guizado-Salazar et al. 2020), advierten sobre la importancia que tiene que, por parte del docente, se entrene con mayor eficacia en procesos pedagógicos, esto tendrá mayor posibilidad de ser efectivos a la hora de fomentar el aprendizaje, siendo 
concordante con la idea de profundizar en la mejora de aplicación del AA en la muestra de estudio.

Por otro lado, la postura de (Tobón-Villada et al. 2021), incluye como aspecto importante de la autorregulación en el estudiante, lo referido al fomento de las inteligencias múltiples como factor complementario a la gestión efectiva del aprendizaje, sobre todo cuando se trabaja bajo la modalidad virtual. Siendo indispensable tener en consideración, el estilo de vida del estudiante (Villavicencio-Guardia et al. 2020), por cuanto este puede influir en la metacognición como proceso inicial en la conformación de un AA efectivo en pro del rendimiento académico, siendo los más desfavorables, aquellos estilos que atentan contra la salud, requiriéndose un auto cuidado para contar con mayor posibilidad de estar saludables, con fines de ser efectivos en el aprendizaje.

Siendo considerable comprender, la importancia que tiene la adaptación de las estrategias del AA de la modalidad presencial a la virtual, con la intención de experimentar su eficacia, aunque esto no es restrictivo, el docente puede configurar nuevas estrategias a partir de la experiencia previa sobre el hecho educativo basado en el enfoque de AA (Berridi-Ramírez \& Martínez-Guerrero, 2017).

\section{CONCLUSIÓN}

El resultado evidenciado en el estadígrafo Rho de Spearman, evidencia una correlación positiva baja o débil (Rho $=0,285)$. La significancia es menor que $0,05(0,000<0,05)$ por lo que se rechaza la hipótesis nula y se acepta la hipótesis alternativa; en este sentido, existe relación entre el aprendizaje autorregulado y rendimiento académico. Se advierte que es necesario promover desde el AA, la autoeficacia académica, siendo un sub proceso que conduce progresivamente a que el estudiante, adopte con mayor responsabilidad su aprendizaje.

\section{FINANCIAMIENTO}

No monetario. 
Revista Arbitrada Interdisciplinaria KOINONIA

Año VI. Vol VI. N³. Edición Especial: Educación II. 2021

Hecho el depósito de Ley: FA2016000010

ISSN: 2542-3088

FUNDACIÓN KOINONIA (F.K). Santa Ana de Coro. Venezuela.

Milagros Yovana Muñoz-Cabana

\section{AGRADECIMIENTO}

A la Universidad Nacional Mayor de San Marcos, Lima; por motivar el desarrollo y fomento de la investigación.

\section{REFERENCIAS CONSULTADAS}

Alegre, A. (2014). Autoeficacia académica, autorregulación del aprendizaje y rendimiento académico en estudiantes universitarios iniciales [Academic self-efficacy, selfregulation of learning and academic performance in initial university students]. Propósitos y Representaciones, 2(1), 79-120. http://dx.doi.org/10.20511/pyr2014.v2n1.54

Barreto-Trujillo, F. J., \& Álvarez-Bermúdez, J. (2020). Estrategias de autorregulación del aprendizaje y rendimiento académico en estudiantes de bachillerato [Selfregulation strategies for learning and academic performance in high school students]. Revista de Estudios e Investigación en Psicología y Educación, 7(2), 184-193. https://doi.org/10.17979/reipe.2020.7.2.6570

Berridi-Ramírez, R, \& Martínez-Guerrero, J. (2017). Estrategias de autorregulación en contextos virtuales de aprendizaje [Self-regulation strategies in virtual learning context]. Perfiles educativos, 39(156), 89-102.

Chambi-Choque, A, Cienfuegos, J, \& Espinoza-Moreno, T. (2020). Estilos de aprendizaje y rendimiento académico en internos de enfermería de una Universidad Pública Peruana [Learning styles and academic performance in nursing interns of a Peruvian Public University]. Revista de la Facultad de Medicina Humana, 20(1), 43-50. https://dx.doi.org/10.25176/rfmh.v20i1.2546

Daura, F. (2015). Aprendizaje autorregulado y rendimiento académico en estudiantes del ciclo clínico de la carrera de Medicina [Self-regulated learning and academic performance in students of the clinical cycle of the Medicine career]. REDIE. Revista Electrónica de Investigación Educativa, 17(3),28-45.

Guizado-Salazar, G, Valenzuela Rodríguez, M, \& Vallejo Quispe, P. (2020). Desempeño docente y el rendimiento académico de los estudiantes de la Facultad de Tecnología en la Universidad Nacional de Educación de Perú [Teaching performance and academic performance of students of the Faculty of Technology at the National University of Education of Peru]. Conrado, 16(72), 200-203.

Hernández, R., Fernández, C., \& Baptista, P. (2014). Metodología de la Investigación [Investigation methodology] (6a. ed.). México: McGraw-Hill. 
Revista Arbitrada Interdisciplinaria KOINONIA

Año VI. Vol VI. N³. Edición Especial: Educación II. 2021

Hecho el depósito de Ley: FA2016000010 ISSN: 2542-3088

FUNDACIÓN KOINONIA (F.K). Santa Ana de Coro. Venezuela.

Milagros Yovana Muñoz-Cabana

Mora-Yate, C. T., Mahecha Escobar, J., \& Conejo Carrasco, F. (2020). Procesos de autorregulación del aprendizaje y desempeño académico en estudiantes de pregrado bajo la modalidad virtual [Self-regulation processes of learning and academic performance in undergraduate students under the virtual modality]. CULTURA EDUCACIÓN Y SOCIEDAD, 11(2), 191-206. https://doi.org/10.17981/cultedusoc.11.2.2020.12

Pintrich, P. (2000). The role of goal orientation in self-regulated learning. En M Boekaerts; P. R. Pintrich y M. Zeidner (Eds.). Handbook of Self-Regulation (pp. 451-502). San Diego: Academic Press.

Rodríguez-Rodríguez, D, \& Guzmán-Rosquete, R. (2019). Rendimiento académico y factores sociofamiliares de riesgo. Variables personales que moderan su influencia [Academic performance and socio-family risk factors. Personal variables that moderate their influence]. Perfiles educativos, 41(164), 118134. https://doi.org/10.22201/iisue.24486167e.2019.164.58925

Tobón-Villada, J. E., Marín Buitrago, O., Tapia Llanos, R. C., \& Martín García, M. A. (2021). Estrategia didáctica de aprendizaje basada en inteligencias múltiples predominantes y procesos autorregulatorios en estudiantes rurales de primaria [Didactic learning strategy based on predominant multiple intelligences and selfregulatory processes in rural primary school students]. INNOVA Research Journal, 6(3), 34-57. https://doi.org/10.33890/innova.v6.n3.2021.1751

Vázquez, S, \& Daura, F. (2013). Auto-regulación del aprendizaje y rendimiento académico [Self-regulation of learning and academic performance]. Estudios pedagógicos (Valdivia), 39(1), 305-324. https://dx.doi.org/10.4067/S0718$\underline{07052013000100018}$

Villavicencio-Guardia, M, Deza y Falcón, I, \& Dávila Soto, R. (2020). Estilos de vida y el rendimiento académico de los estudiantes de una facultad de enfermería en Perú [Lifestyles and academic performance of students of a nursing school in Peru]. Conrado, 16(74), 112-119.

(O2021 por los autores. Este artículo es de acceso abierto y distribuido según los términos y condiciones de la licencia Creative Commons Atribución-NoComercial-Compartirlgual 4.0 Internacional (CC BY-NC-SA 4.0) (https://creativecommons.org/licenses/by-nc-sa/4.0/). 